\title{
Cooperative facility location games
}

\author{
Michel X. Goemans ${ }^{1,3} \quad$ Martin Skutella ${ }^{2,3}$
}

\begin{abstract}
The location of facilities in order to provide service for customers is a well-studied problem in the operations research literature. In the basic model, there is a predefined cost for opening a facility and also for connecting a customer to a facility, the goal being to minimize the total cost. Often, both in the case of public facilities (such as libraries, municipal swimming pools, fire stations, ...) and private facilities (such as distribution centers, switching stations, ...), we may want to find a 'fair' allocation of the total cost to the customers - this is known as the cost allocation problem. A central question in cooperative game theory is whether the total cost can be allocated to the customers such that no coalition of customers has any incentive to build their own facility or to ask a competitor to service them.

We establish strong connections between fair cost allocations and linear programming relaxations for several variants of the facility location problem. In particular, we show that a fair cost allocation exists if and only if there is no integrality gap for a corresponding linear programming relaxation; this was only known for the simplest unconstrained variant of the facility location problem. Moreover, we introduce a subtle variant of randomized rounding and derive new proofs for the existence of fair cost allocations for several classes of instances. We also show that it is in general NP-complete to decide whether a fair cost allocation exists and whether a given allocation is fair.
\end{abstract}

Keywords: facility location, cooperative games, LP relaxation, randomized rounding, core

MSC: 91A12, 90B80, 90C35, 90C90

\section{Introduction}

Fair cost allocations. In the facility location problem, customers from a given set $N$ are in need of a certain service which can be provided by connecting them to facilities. These facilities could be railway stations, sports complexes, libraries, switching stations, antennas, or supermarkets, to cite a few examples. From a given set $F$ of possible locations for the facilities, one has to decide first which facilities to open (build) and then each customer must be assigned (connected) to an open facility. Opening facility $i \in F$ causes a fixed cost $f_{i} \geqslant 0$ and the cost for connecting customer $j \in N$ to this facility is denoted by $c_{i j} \geqslant 0$. We refer to this problem as the unconstrained (or uncapacitated) facility location problem; this problem is also sometimes referred to as the uncapacitated plant location problem.

In many situations, further constraints have to be taken into consideration. The facilities can typically handle only a limited number of customers, say at most $k_{i}$ customers for facility $i$. Certain customers cannot be assigned to certain facilities (for example, if they are geographically too far apart from each other); this can be handled in the original unconstrained model by simply letting the corresponding $\cos t c_{i j}$ be very large (or infinite). Other relevant constraints can occur when the set of customers is heterogeneous and some quota must be met: At least some fraction of the customers connected to a facility must belong to a certain subgroup or minority (e.g., at least $40 \%$ walloons, $33 \%$ socio-democrats, an equal number of women and men, etc.). Or, members of different groups cannot be assigned to the same facility (e.g., each facility represents a factory that can produce only one product and customers request one of the different products).

From a central authority's point of view, it is interesting to ask for a cheapest possible solution, i. e., to minimize the total cost which is made up by the cost to build facilities and to connect the customers to the open facilities.

\footnotetext{
${ }^{1}$ Department of Mathematics, Room 2-351, M.I.T., Cambridge, MA 02139, USA. Email: goemans@math .mit .edu. Supported in part by NSF contract $9623859-\mathrm{CCR}$.

${ }^{2}$ TU Berlin, Fakultät II, Institut für Mathematik, MA 6-1, Straße des 17. Juni 136, 10623 Berlin, Germany. Email: skutel la@math . tuberlin. de. Supported by DONET within the frame of the TMR Programme (contract number ERB FMRX-CT98-0202).

${ }^{3}$ The research was performed when the authors were at C.O.R.E., Louvain-la-Neuve, Belgium.
} 
This facility location problem has attracted much attention in the operations research literature, see for example the book Mirchandani and Francis (1990).

Facility location games. We can also ask whether the total cost can be allocated to the different customers in a fair way. This is known as the cost allocation problem. For example, towns would pay for the building of libraries, or sports complexes, but they don't want to pay more than their fair share of the total cost, whatever that means. In the area of cooperative game theory, see for example Moulin (1995), fairness means that no group of customers, or coalition, has any incentive to break apart and obtain the service on their own. In other words, if $v_{j}$ denotes the price being paid by customer $j$, we would like that $\sum_{j \in S} v_{j} \leqslant \operatorname{OPT}(S)$ where $S$ is any subset of customers and $\operatorname{OPT}(S)$ represents the cost of providing the service only to the customers in $S$. The core of the cooperative game is then defined as

$$
\begin{array}{rlrl}
\text { core }=\left\{v: \sum_{j \in N} v_{j}\right. & =\operatorname{OPT}(N) & \\
\sum_{j \in S} v_{j} \leqslant \operatorname{OPT}(S) & \text { for all } S \subseteq N\}
\end{array}
$$

and a central question in cooperative game theory is whether the core is non-empty, and if so, how to find an allocation vector in the core. Traditionally, the non vacuity of the core is established by showing that the game is balanced (for definitions, see Moulin (1995)). In linear programming terms, this boils down to showing that any extreme point of the dual to $\max \sum_{j \in N} v_{j}$ subject to (1) has value at least $\operatorname{OPT}(N)$. It is well known and easy to check that, for a submodular function $\operatorname{OPT}(\cdot)$ (i. e., $\operatorname{OPT}(S)+\operatorname{OPT}(T) \geqslant \operatorname{OPT}(S \cup T)+\operatorname{OPT}(S \cap T)$ for all $S, T \subseteq N)$, the core is non-empty and the Shapley value (cf. Moulin (1995)) lies in the core. However, it is also known that this condition is in general not satisfied for the facility location game under consideration. In Figure 1 we give a facility location instance for which the function $\operatorname{OPT}(\cdot)$ is not submodular; nevertheless, the core is non-empty in this example.

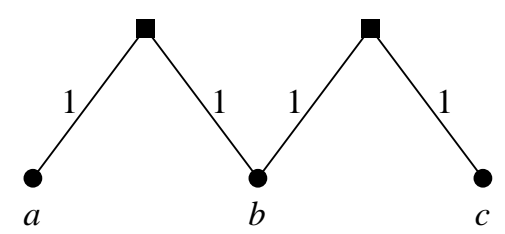

Figure 1: An instance with two facilities (squares) and three customers $a, b$ and $c$ (circles). The cost for opening a facility is 1 and the connection costs are given by the distances in the drawn graph. Notice that the function OPT $(\cdot)$ is not submodular since $\operatorname{OPT}(\{a, b, c\})+\operatorname{OPT}(\{b\})>\operatorname{OPT}(\{a, b\})+\operatorname{OPT}(\{b, c\})$. The unique element in the core is given by $v_{a}=v_{c}=2$ and $v_{b}=1$.

Related LP relaxations. When the core is empty, we would like instead to recover as much as possible and maximize $\sum_{j \in N} v_{j}$ subject to the constraints (1). Observe that this is a linear program (LP) but the constraints defining it are not only exponential in number but also not well characterized since the right-hand-side value $\operatorname{OPT}(S)$ is an NP-hard quantity for general facility location problems. However, the value of this linear program is a lower bound on the optimum value $\operatorname{OPT}(N)$ and thus it can be viewed as a relaxation of the problem.

In this paper, for any kind of constrained facility location problem, we show how to derive an equivalent relaxation in the natural space of variables which contains a variable $y_{i}$ denoting whether facility $i$ is open and a variable $x_{i j}$ denoting whether customer $j$ is assigned to facility $i$. In general, our result thus says that the core is nonempty if and only if this canonical LP relaxation has no integrality gap for the objective function being considered, i. e., the optimum LP value is equal to $\operatorname{OPT}(N)$. This result and the canonical LP relaxation are described in Section 2.

For the unconstrained facility location problem, this canonical relaxation turns out to simply be a classical LP relaxation of the problem, a result first derived by Kolen (1983). Kolen shows that the dual of this classical LP relaxation for the uncapacitated case can be interpreted as the maximum amount that can be allocated to the customers subject to the constraints (1) and, as a result, the core is non-empty if and only if there is no integrality gap for this relaxation. Our approach is similar and allows to derive (implicitly, and in some cases, 
explicitly) this canonical relaxation for any facility location problem. Chardaire (1998) also considers the classical LP relaxation of the uncapacitated facility location problem and shows that the associated game can be viewed as a linear production game that satisfies Owen's additivity assumption (Owen 1995). As a result, he derives that the core of this linear programming game is non-empty, and hence the core of the original uncapacitated facility location game is non-empty if the LP relaxation has no integrality gap. Furthermore, he uses Kolen's result to derive the converse statement. Chardaire also generalizes some of his results to variants of the capacitated facility location problem but does not characterize the canonical relaxation whose absence of integrality gap is a necessary and sufficient condition for non emptiness of the core. We refer the reader to Chardaire for a discussion of related work of Sharkey (1990) on a similar revenue game and of Göthe-Lundgren, Jörnsten, and Värbrand (1996) on a set partitioning game.

Connections between the core and certain LP relaxations have also been found for other cooperative games related to problems in combinatorial optimization. Deng, Ibaraki, and Nagamochi (1999) consider general packing and covering games given through integer linear programs (ILP) with a $\{0,1\}$-matrix and right hand side all ones; they show that the core of such a game is non-empty if and only if the natural LP relaxation of the ILP has an integer optimal solution. Moreover, they give applications of this general result for various games on graphs related to wellknown combinatorial optimization problems, such as maximum flow, maximum matching, coloring, and others; see also Deng, Ibaraki, Nagamochi, and Zang (2000). The result of Kolen (1983) on the unconstrained facility location game can also be derived from this more general framework since the unconstrained facility location problem can be formulated as such a covering problem, see, e. g., Ageev (1983) (cited in Grishukhin (1994)) or Kolen and Tamir (1990). Further results in this direction have for example been derived by Faigle and Kern (2000) and Samet and Zemel (1984).

In contrast to the earlier results mentioned above, we develop our results and techniques in a setting which allows us to handle not only the unconstrained facility location problem but also arbitrarily constrained variants of it. Moreover, in comparison to Kolen (1983) and Chardaire (1998), our proof is more straightforward. For general facility location problems, we can in certain cases give an explicit representation of the canonical relaxation in terms of linear inequalities. However, even if we are unable to completely characterize the relaxation in terms of linear inequalities, we can nevertheless find a fair allocation that maximizes the amount recovered provided that we can find the best feasible assignment of customers to a single facility. This is discussed in Section 3.

Non-emptiness of the core for special cases. In Section 4, we provide new proofs showing that the core of the unconstrained facility location game is non-empty for two special cases, one in which the facilities can be ordered on a line and the connection costs are unimodal (i.e., first decreasing and then increasing), and the other when the facilities are positioned on a tree and the connection costs are obtained by applying a nondecreasing function (depending on the customer) to the tree metric. These results have been obtained earlier by Trubin (1976) (see also Grishukhin (1994)) and also by Kolen (1983) (see also Kolen and Tamir (1990) and Cornuéjols, Nemhauser, and Wolsey (1990)).

Our main contribution in this section is a different proof technique. We use randomized rounding to show that the canonical relaxation has no integrality gap for these special classes of instances. The randomized rounding is performed in a dependent way by assigning subsets of $[0,1)$ to each facility and to each connection of customers to facilities. This algorithmic proof technique is of independent interest. For example, it can be applied to simplify a recent result of Bar-Noy, Guha, Naor, and Schieber (1999). Or it can be used to derive the result of Shmoys and Tardos (1993) on the generalized assignment problem without the need for explicitly solving a matching problem, in a way more similar to Lenstra, Shmoys, and Tardos (1990). For the case of facility location on a line with unimodal connection costs, our randomized rounding approach is closely related to a technique introduced by Bertsimas, Teo, and Vohra (1999) to prove the integrality of an LP formulation of the uncapacitated lot-sizing problem.

Complexity results. In Section 5, we show that even for the unconstrained facility location problem, testing whether there is an integral optimal solution (or no integrality gap) to the canonical LP relaxation is actually NPcomplete. Our proof also yields that checking whether a given cost allocation is in the core is an NP-complete problem. On the other hand, if the core is known to be non-empty, the latter problem can be solved in polynomial time and an element of the core can also be computed in polynomial time.

Deng, Ibaraki, and Nagamochi (1999) derive similar results for different games on graphs (see Table 1 in their paper); surprisingly, there seems to be no direct correlation between the complexity of those questions for cooperative games and the complexity of the corresponding combinatorial optimization problems. Further results 
in this direction have been obtained, e. g., by Deng and Papadimitriou (1994) and by Faigle, Fekete, Hochstättler, and Kern (1997).

Known results on the size of the integrality gap. Since for the uncapacitated facility location problem the optimal value of the classical LP relaxation is equal to the maximum amount that can be recovered in the cost allocation problem, results on the worst case ratio of the integrality gap gain a new meaning in the context of cost allocation. It follows for example from the LP-based approximation result of Chudak (1998) that, for metric instances (i.e., when the costs $c_{i j}$ arise from a metric on $N \cup F$ ), there always exists a fair cost allocation that recovers at least a fraction $e /(e+2) \approx 0.576$ of the total cost. On the other hand, Guha and Khuller (1998) give a class of metric instances where at most $68 \%$ of the total cost can be recovered. This gives worst-case bounds on the amount that the central authority should subsidize in order to ensure the existence of a fair allocation. However, for general cost functions, there exist instances for which the amount one can recover is at $\operatorname{most} \operatorname{OPT}(N) \frac{2 n}{n+1} \frac{1}{\log _{2}(n+1)}$ (using a standard reduction from the set cover problem and using instances with large integrality gaps for the set cover problem, see Vazirani (1999)).

Extension to a closely related problem. A problem that is closely related to the facility location problem occurs when connections of customers to facilities do not cause costs $c_{i j}$ but produce certain non-negative benefits $b_{i j}$. Here, a customer can be connected to at most one facility and the goal is to maximize the total benefit minus the cost for building facilities. In the corresponding cooperative game we ask for a fair allocation of this amount to the customers, i. e., each coalition of customers wants to get at least as much as it could gain on its own. The results in this paper can easily be carried forward to this setting.

\section{Integer and linear programming formulations}

In order to model the facility location problem we introduce two types of binary variables: For each $i \in F$, the variable $y_{i}$ is 1 if facility $i$ is opened and 0 otherwise; for each $i \in F$ and $j \in N$, the variable $x_{i j}$ is 1 if customer $j$ is connected to facility $i$ and 0 otherwise. A minimum cost solution to the basic version of the facility location problem is then given by the following integer linear program:

$$
\begin{array}{lll}
\text { minimize } & \sum_{i \in F} f_{i} y_{i}+\sum_{\substack{i \in F \\
j \in N}} c_{i j} x_{i j} & \\
\text { subject to } & \sum_{i \in F} x_{i j}=1 & \text { for all } j \in N, \\
& y_{i}-x_{i j} \geqslant 0 & \text { for all } i \in F, j \in N, \\
& x_{i j}, y_{i} \in\{0,1\} & \text { for all } i \in F, j \in N .
\end{array}
$$

Constraints (2) ensure that every customer is connected to exactly one facility. A customer can only be connected to a facility that is open by constraints (3). For the capacitated version of the problem we add the following capacity constraints:

$$
k_{i} y_{i}-\sum_{j \in N} x_{i j} \geqslant 0 \quad \text { for all } i \in F
$$

If at least a fraction $q_{i}$ of the customers connected to facility $i$ have to belong to a subgroup $N^{\prime} \subseteq N$, we add the quota constraints:

$$
\left(1-q_{i}\right) \sum_{j \in N^{\prime}} x_{i j}-q_{i} \sum_{j \in N \backslash N^{\prime}} x_{i j} \geqslant 0 \quad \text { for all } i \in F
$$

If the set of customers is partitioned into subsets $N_{p}, p=1, \ldots, l$, and a facility can only serve customers in at most one subset, we add the incompatibility constraints:

$$
x_{i j}+x_{i k} \leqslant y_{i} \quad \text { for all } i \in F \text {, all } j \in N_{p} \text { and all } k \in N_{q}, p \neq q .
$$

Notice that constraints (4), (5) and (6) do not introduce a coupling between different facilities but can be expressed solely in terms of the variables $x_{i j}$ and $y_{i}$ for each fixed facility $i$. We consider a more general class of constraints 
where, for each facility $i$, we are given a family of subsets $S \subseteq N$ of customers that can be connected to this facility. In this case we can rewrite the integer program as: minimize $\sum_{i} f_{i} y_{i}+\sum_{i, j} c_{i j} x_{i j}$ subject to $\sum_{i} x_{i j}=1$ for all $j \in N$ and $\left(y_{i}, x_{i}\right.$. $) \in P_{i}$ for all $i \in F$. Here, $x_{i} .:=\left(x_{i 1}, \ldots, x_{i n}\right)$, with $n:=|N|$, and $P_{i} \subseteq\{0,1\}^{n+1}$ is given by

$$
P_{i}:=\{(0, \ldots, 0)\} \cup\left\{\left(1, \chi_{S}\right): S \subseteq N \text { feasible for } i\right\}
$$

where $\chi_{S} \in\{0,1\}^{n}$ denotes the characteristic vector of the subset $S$.

There are several possible ways of deriving a linear programming relaxation for this problem. The most natural would be to try to replace each discrete set $P_{i}$ by its convex hull $\operatorname{conv}\left(P_{i}\right)$. Notice that the value of the resulting linear program might not be equal to OPT $(N)$ since the intersection of the convex hulls with the hyperplanes (2) is not necessarily the convex hull of the intersections. A slightly weaker relaxation would be to replace each $P_{i}$ by its conic hull cone $\left(P_{i}\right)=\left\{\sum_{x \in P_{i}} \lambda_{x} x: \lambda_{x} \geqslant 0\right\}$. Given the special form of $P_{i}$, it is easy to see that $\operatorname{conv}\left(P_{i}\right)=$ cone $\left(P_{i}\right) \cap\{(y, x): y \leq 1\}$. This leads to the following relaxation (LP):

$$
\begin{array}{lll}
\text { minimize } & \sum_{i \in F} f_{i} y_{i}+\sum_{\substack{i \in F \\
j \in N}} c_{i j} x_{i j} & \\
\text { subject to } & \sum_{i \in F} x_{i j}=1 & \text { for all } j \in N, \\
& \left(y_{i}, x_{i}\right) \in \operatorname{cone}\left(P_{i}\right) & \text { for all } i \in F .
\end{array}
$$

We now turn to the cost allocation problem. For each coalition $S \subseteq N$, let OPT $(S)$ denote the minimum cost of the facility location problem restricted to the set of customers $S$. The maximum cost that can be allocated to the customers is then given by the following linear program (CAP):

$$
\begin{array}{lll} 
& \text { maximize } & \sum_{j \in N} v_{j} \\
\text { (CAP) } \quad \text { subject to } & \sum_{j \in S} v_{j} \leqslant \operatorname{OPT}(S) \quad \text { for all } S \subseteq N .
\end{array}
$$

It is an easy observation that the amount $v_{j}$ that is paid by customer $j$ in an optimal cost allocation is always nonnegative (since $c_{i j} \geqslant 0$ implies $\mathrm{OPT}(S) \leqslant \mathrm{OPT}(S \cup\{j\})$ ). Although there are exponentially many constraints and although it is in general NP-hard to compute the right hand side of this linear program, we show that in some cases it can be solved in polynomial time. To obtain this result we develop the following connection to the LP relaxation of the facility location problem introduced above.

Theorem 2.1. The cost allocation problem (CAP) is equivalent to the dual of the LP relaxation $(L P)$ of the facility location problem. In particular, their values are equal and the core is non-empty if and only if there is no integrality gap for the relaxation $(L P)$ of the facility location problem.

Proof. We dualize constraints (7) and introduce a vector $v$ of corresponding dual variables $v_{j}$ for all customers $j \in N$. This leads to the following program of the same value as (LP) by strong duality; see, e. g., (Nemhauser and Wolsey 1988, Section II.3.6.):

$$
\begin{array}{lll}
\max _{v} \min _{x, y} & \sum_{i \in F} f_{i} y_{i}+\sum_{\substack{i \in F \\
j \in N}}\left(c_{i j}-v_{j}\right) x_{i j}+\sum_{j \in N} v_{j} & \\
\text { subject to } & \left(y_{i}, x_{i} .\right) \in \operatorname{cone}\left(P_{i}\right) & \text { for all } i \in F .
\end{array}
$$

For a fixed vector $v$, the program is decomposed into the sum of $n=|N|$ linear minimization problems over pointed cones. Therefore, the inner minimization problem is either unbounded or an optimum solution is given by $x_{i j}=y_{i}=0$, for all $i \in F, j \in N$, and has value $\sum_{j} v_{j}$. Moreover, since cone $\left(P_{i}\right)$ is generated by the incidence vectors of feasible assignments to facility $i$, the inner minimization problem is unbounded if and only if there exists an $i \in F$ and a corresponding feasible coalition $S \subseteq N$, i. e., $\left(1, \chi_{S}\right) \in P_{i}$, with

$$
f_{i}+\sum_{j \in S}\left(c_{i j}-v_{j}\right)<0 .
$$


Thus, we can rewrite the program as:

$$
\begin{array}{ll}
\text { maximize } & \sum_{j \in N} v_{j} \\
\text { subject to } & \sum_{j \in S} v_{j} \leqslant f_{i}+\sum_{j \in S} c_{i j} \quad \text { for all } i \in F \text { and } S \subseteq N \text { feasible. }
\end{array}
$$

Since the right hand side of constraints (8) is stronger than the right hand side of (9), it remains to show that (8) is implicitly contained in (9). Suppose that an optimal solution to the subproblem induced by $S$ is given by

$$
\operatorname{OPT}(S)=\sum_{i \in F^{\prime}}\left(f_{i}+\sum_{j \in S_{i}} c_{i j}\right)
$$

where $F^{\prime} \subseteq F$ and the $S_{i}, i \in F^{\prime}$, form a partition of $S$. Now observe that (8) can be obtained by simply aggregating constraints (9) over $i \in F^{\prime}$ for $S=S_{i}$. This completes the proof.

Let us turn to describing cone $\left(P_{i}\right)$ for a few special cases. For the unconstrained facility location problem, the conic hull of the set $P_{i}$ is given by cone $\left(P_{i}\right)=\left\{\left(y_{i}, x_{i}\right): 0 \leqslant x_{i j} \leqslant y_{i}\right.$ for all $\left.j \in N\right\}$; this yields the following classical LP relaxation which has been introduced by Balinski (1965):

$$
\begin{array}{cll}
\text { minimize } & \sum_{i \in F} f_{i} y_{i}+\sum_{\substack{i \in F \\
j \in N}} c_{i j} x_{i j} & \\
\text { subject to } & \sum_{i \in F} x_{i j}=1 & \text { for all } j \in N, \\
& 0 \leqslant x_{i j} \leqslant y_{i} & \text { for all } i \in F, j \in N .
\end{array}
$$

Notice that there exists an optimal solution to this LP relaxation with $y_{i} \leqslant 1$ for all $i \in F$. In this case, having replaced $P_{i}$ by cone $\left(P_{i}\right)$ rather than $\operatorname{conv}\left(P_{i}\right)$ does not matter. This is no longer true if we turn to the capacitated version of the facility location problem including constraints (4); in this case we get

$$
\text { cone }\left(P_{i}\right)=\left\{\left(y_{i}, x_{i}\right): \sum_{j} x_{i j} \leqslant k_{i} y_{i} \text { and } 0 \leqslant x_{i j} \leqslant y_{i} \text { for all } j \in N\right\}
$$

such that we only have to add the constraints (4) to the above LP relaxation.

Consider an example with two facilities of capacity 1 and fixed cost 0 and two customers that are located at the first facility; they can be connected to this facility for free while the connection to the second facility costs 1 . In an optimal solution one of the customers has to be connected to the second facility which causes cost 1 ; however, an optimal LP solution has value 0 since it can open the first facility with $y_{1}=2$ and connect both customers to this facility. If we add the constraint $y_{1} \leqslant 1$ to the LP relaxation, its optimum value increases to 1 .

In fact, in this example, none of the customers is willing to pay anything for the service since he can argue that he could connect to the first facility for free. Therefore, the cost allocation problem is equivalent to the dual of the weak LP relaxation but not to the dual of the stronger relaxation including constraints $y_{i} \leqslant 1$ for all $i \in F$, which would have been obtained if we had relaxed $P_{i}$ to $\operatorname{conv}\left(P_{i}\right)$. This answers an open question discussed by Chardaire (1998).

If, instead of capacity constraints, we have that each facility must serve the same number of customers from $N_{1}$ and from $N_{2}$, then we simply have that:

$$
\operatorname{cone}\left(P_{i}\right)=\left\{\left(y_{i}, x_{i .}\right): \sum_{j \in N_{1}} x_{i j}=\sum_{j \in N_{2}} x_{i j} \text { and } 0 \leqslant x_{i j} \leqslant y_{i} \text { for all } j \in N\right\} .
$$

If we have incompatibility constraints $x_{i j}+x_{i k} \leq y_{i}$ for certain pairs $(j, k) \in E_{i}$ as in (6), we need to include all inequalities that describe (the conic version of) the stable set polytope for the graph $\left(N, E_{i}\right)$. For the specific form (6), the corresponding graph is a complete $l$-partite graph, and therefore the clique constraints

$$
\sum_{p} x_{i j(p)} \leq y_{i} \quad \text { for all }(j(1), j(2), \ldots, j(l)) \in N_{1} \times N_{2} \times \ldots \times N_{l}
$$

are sufficient since the graph is perfect; see Grötschel, Lovász, and Schrijver (1988).

However, if we consider quota constraints (5) or we combine simultaneously say capacity constraints (4) and incompatibility constraints (6), then additional more complicated inequalities are needed to describe the conic hull. 


\section{Polynomial-time solvability of the cost allocation problem}

As a result of Theorem 2.1, the cost allocation problem (CAP) can be solved in polynomial time by linear programming if we know a compact (i. e., with a polynomial number of linear inequalities) representation of the cones generated by the $P_{i}$ 's. In this case, an element of the core can simply be obtained by solving (LP) and extracting the dual variables on the constraints (7).

Even if we don't know or there doesn't exist a compact (possibly extended) representation of each cone, we can still solve the cost allocation problem in polynomial time provided we can optimize in polynomial time over each discrete set $P_{i}$. This can be done by using the ellipsoid method and exploiting the equivalence between optimization and separation, see Grötschel, Lovász, and Schrijver (1988). Since we have a polynomial bound on the size of the inequalities needed to describe (CAP), the equivalence is between strong (or exact) optimization and separation. The separation problem associated to (CAP) is given a vector $v$ to decide whether there exists a set $S \subseteq N$ such that $\sum_{j \in S} v_{j}>\operatorname{OPT}(S)$, and if so to find one such set. By the same reasoning as in the proof of Theorem 2.1, this is equivalent to deciding whether there exists $i \in F$ and a feasible $S$ for $i$ such that

$$
f_{i}+\sum_{j \in S}\left(c_{i j}-v_{j}\right)<0 .
$$

And this can be decided by optimizing over $P_{i}$.

Similarly if we have a polynomial approximation scheme for the $P_{i}$ 's then we can derive a polynomial approximation scheme for (CAP). This is for example useful when the customers have different demands, say $d_{j}$ for customer $j$, and we have a capacity constraint on each facility:

$$
\sum_{j} d_{i} x_{i j} \leqslant k_{i} y_{i}
$$

\section{On the existence of core elements}

In the literature, positive and negative results on the existence of elements in the core have been shown for several important classes of instances. In view of the result in Theorem 2.1, we provide a novel variant of randomized rounding in order to give new and simple proofs for the existence of integral solutions for the LP relaxation of facility location problems in certain cases. Randomized rounding is a well known technique in combinatorial optimization for turning a fractional solution into an integer solution making use of the structural information contained in the fractional solution; we refer the reader to Motwani and Raghavan (1995) for further information.

For the unconstrained facility location problem, we take an optimum solution $(x, y)$ to the LP relaxation discussed in Section 2 and try to round it randomly to a feasible integral solution by interpreting the fractional values $x_{i j}$ and $y_{i}$ as probabilities. A similar technique was used by Chudak (1998) to compute near-optimal solutions for metric instances. However, while Chudak opened facilities randomly with probabilities $y_{i}$ but established connections by a different routine, the main problem for our approach is to make sure that a variable $x_{i j}$ is only rounded to 1 if facility $i$ is open, $i$. e., if the variable $y_{i}$ is also rounded to 1 . This condition forces a coupling of the random decisions which makes it necessary to introduce a subtle correlation between the different random variables.

We first 'color' all facilities $i \in F$ by subsets $I_{i}$ of the interval $[0,1)$ such that the measure $\left|I_{i}\right|$ of $I_{i}$ is equal to $y_{i}$. Later, we will draw a random variable $\alpha$ uniformly distributed from $[0,1)$ and open all facilities $i$ with $\alpha \in I_{i}$; in fact, the probability for opening facility $i$ is then equal to $y_{i}$. In order to determine the connections of customers $j$ to facilities $i$, we construct subsets $I_{i j}$ of $[0,1)$ such that $\left|I_{i j}\right|=x_{i j}$ and establish a connection from customer $j$ to facility $i$ if $\alpha \in I_{i j}$. To make sure that each customer is connected to exactly one facility, the subsets $I_{i j}, i \in F$, should form a partition of the interval $[0,1)$; notice that $\sum_{i \in F}\left|I_{i j}\right|=\sum_{i \in F} x_{i j}=1$ by constraints (10). Moreover, since a customer should only be connected to an open facility, we require $I_{i j} \subseteq I_{i}$ for all $i \in F, j \in N$.

Lemma 4.1. Given an optimum solution $(x, y)$ to the LP relaxation of the unconstrained facility location problem, if we can find subsets $I_{i}$ and $I_{i j}$ of $[0,1)$ with the following properties:

i) $\left|I_{i}\right|=y_{i},\left|I_{i j}\right|=x_{i j}$, for all $i \in F, j \in N$;

ii) $\bigcup_{i \in F} I_{i j}=[0,1)$, for all $j \in N$, and $I_{i j} \cap I_{i^{\prime} j}=\emptyset$, for all $i \neq i^{\prime} \in F, j \in N$;

iii) $I_{i j} \subseteq I_{i}$, for all $i \in F, j \in N$; 
then there exists an integral optimal solution to the LP relaxation.

Proof. We randomly construct an integral solution $\left({ }^{-} x,-\bar{y}\right)$ : Choose a random variable $\alpha$ uniformly distributed from $[0,1)$; open all facilities $i$ with $\alpha \in I_{i}$ (i. e., set ${ }^{-} y=1$ ) and establish all connections $i j$ with $\alpha \in I_{i j}$ (i. e., set ${ }^{-} x_{j}=1$ ). By the properties of the sets $I_{i}$ and $I_{i j}$, this gives a feasible integral solution of expected value

$$
\mathrm{E}\left[\sum_{i \in F} f_{i}{ }^{-} y+\sum_{\substack{i \in F \\ j \in N}} c_{i j}{ }^{-} x_{j}\right]=\sum_{i \in F} f_{i} y_{i}+\sum_{\substack{i \in F \\ j \in N}} c_{i j} x_{i j}=\mathrm{OPT}_{\mathrm{LP}} .
$$

This expected value is a convex combination of the values of all integral solutions corresponding to possible choices of $\alpha$. In particular, there exists an integral solution whose value is upper bounded by the optimum LP value.

\subsection{Facility location on a line with unimodal connection costs}

We apply this proof technique to unconstrained facility location problems where all facilities can be ordered in such a way that, for any customer $j$, the connection costs $c_{i j}$ are unimodal as a function of $i$. This means that there exists an ordering $1, \ldots, m$ of the facilities and for any customer $j$, there exists a facility $i(j)$ such that $c_{i j}$ is nonincreasing for $i \leqslant i(j)$ and nondecreasing for $i \geqslant i(j)$. This is for example the case when all facilities are located on a line in the plane or a higher dimensional Euclidean space and the connection cost between customer $j$ and facility $i$ is a nondecreasing function of their Euclidean distance (see Figure 2). As an illustration, this situation

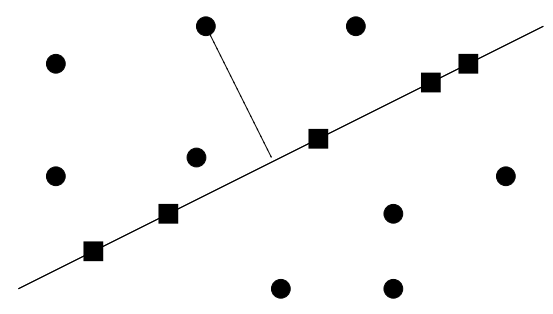

Figure 2: The unimodal case with facilities on a line; the rectangles represent facilities while the circles correspond to customers.

occurs when we have a (straight) railway line and the problem is to decide where to build railway stations so as to provide an optimal service to the inhabitants of the region around the railway line.

Theorem 4.2 (Trubin (1976), Kolen (1983)). There is no integrality gap for the unconstrained facility location problem with unimodal connection costs; in particular, the core is non-empty in this case.

Proof. In the following we assume without loss of generality that the optimal LP solution $(x, y)$ fulfills $y_{i} \leqslant 1$, for all $i \in F$, and has the following property: If facility $i$ lies between facility $i(j)$ and facility $i^{\prime}$ and is distinct from $i^{\prime}$ (i. e., $i(j) \leqslant i<i^{\prime}$ or $\left.i^{\prime}<i \leqslant i(j)\right)$ and $x_{i^{\prime} j}>0$, then $x_{i j}=y_{i}$. Otherwise one can modify the solution accordingly by increasing $x_{i j}$ and simultaneously decreasing $x_{i^{\prime} j}$ without an increase in cost since $c_{i j} \leqslant c_{i^{\prime} j}$. In particular, this implies that $x_{i(j) j}=y_{i(j)}$ for all $j \in N$.

Let $a_{0}:=0$ and $a_{i}:=\sum_{k=1}^{i} y_{k}$, for $i=1, \ldots, m$, and assign the set $I_{i}:=\left(\left[a_{i-1}, a_{i}\right) \bmod 1\right) \subseteq[0,1)$ to facility $i$. Notice that by construction $\left|I_{i}\right|=y_{i}$. We also assign to each pair formed by a facility $i$ and a customer $j$ a subset of measure $x_{i j}$ :

$$
I_{i j}:= \begin{cases}{\left[a_{i}-x_{i j}, a_{i}\right) \quad \bmod 1} & \text { if } i \leqslant i(j), \\ {\left[a_{i-1}, a_{i-1}+x_{i j}\right) \quad \bmod 1} & \text { if } i \geqslant i(j) .\end{cases}
$$

Notice that $I_{i(j) j}=\left[a_{i(j)-1}, a_{i(j)}\right)=I_{i(j)}$ since $x_{i(j) j}=y_{i(j)}$. The fact that $x_{i j} \leqslant y_{i}$ implies that $I_{i j} \subseteq I_{i}$. Moreover, by our assumption on the LP solution $(x, y)$, the subsets $I_{i j}, i \in F$, form a partition of the interval $[0,1)$ for each fixed customer $j$. Therefore, the sets $I_{i}$ and $I_{i j}$ fulfill the conditions of Lemma 4.1 and the result follows.

Notice that in the unimodal case the cost functions for the connections along the line are not necessarily symmetric around $i(j)$. An important application with non-symmetric connection costs is the lot sizing problem: 
The line represents a time axis and facilities and customers correspond to discrete points in time when a product can be produced and has to be delivered, respectively. In particular, if we don't allow backlogging, a customer can only be served by a facility 'in the past', i. e., on its left hand side on the line. In this case, Theorem 4.2 was proved by Krarup and Bilde (1977).

The result in Theorem 4.2 cannot be generalized to the capacitated version of the problem. This follows from the example discussed in Section 2 that can obviously be realized on a line and where the customers are not willing to pay anything although the cost of an optimum solution is positive.

In the case where the facilities are located on a line and each facility must serve the same number of customers from $N_{1}$ and from $N_{2}$, the core can also be empty. Consider the example given in Figure 3 where the same numbers of women and men have to be served by each facility. The fixed cost for opening a facility is 1 . The cost for

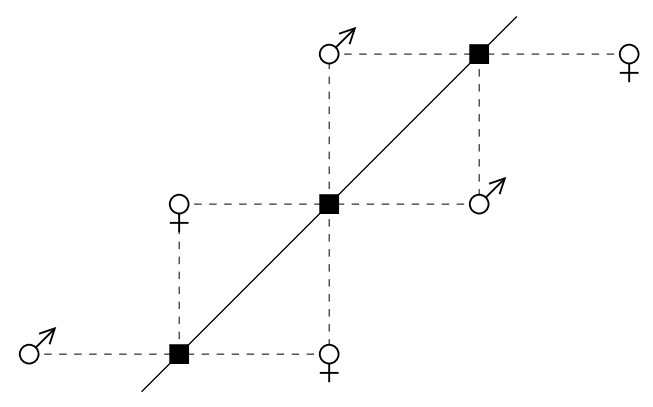

Figure 3: An instance of the facility location problem on a line where each facility has to serve the same number of women and men; the core is empty.

connecting a customer to a facility is equal to the corresponding distance (number of edges on the shortest path) in the graph given by the dotted edges. In an optimal solution, all facilities are opened resulting in total cost 9. However, in the LP relaxation we can open the facility in the middle with fraction $1 / 2$ and also connect the neighboring customers with fraction $1 / 2$; the optimal LP value is therefore only $17 / 2$. It also follows from this example that in the more general case of quota constraints (5) the core can be empty.

\subsection{Facility location on a cycle}

Another possible direction for generalizing the result in Theorem 4.2 is to switch to more complicated topologies than the line. However, it has been observed by Tamir (1992, Example 1) (see also Kolen and Tamir (1990, Example 6.3)) that already instances defined on a cycle with unimodal connection costs can have an empty core; we say that the connection costs on a cycle are unimodal if, for each customer $j$, opening the cycle at a facility $i$ with maximum $c_{i j}$ yields an unimodal cost function on the resulting line. In the example given in Figure 4 the

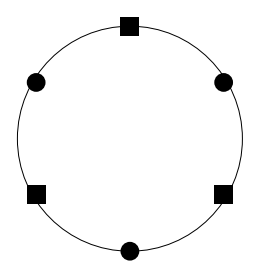

Figure 4: An instance of the facility location problem on a cycle with empty core.

fixed cost for opening each facility is 2 and the connection cost between a customer and a facility is equal to their distance in the cyclic graph. An optimal solution opens two facilities and has total cost 7 . However, the optimum solution to the LP relaxation opens each facility with fraction $1 / 2$ and has value 6 . Notice that the sum of the $y_{i}$ 's is equal to $3 / 2$ and thus not integral in this case.

Theorem 4.3. If there exists an optimum solution to the $L P$ relaxation with $\sum_{i \in F} y_{i} \in \mathbb{Z}$, then there is no integrality gap for the unconstrained facility location problem with unimodal connection costs on a cycle; in particular, the core is non-empty in this case. 
Proof. The proof is a straightforward generalization of the proof of Theorem 4.2. We assume that the facilities $1, \ldots, m$ are ordered clockwise along the cycle and define the sets $I_{i}$ exactly as in the proof of Theorem 4.2. Notice that $a_{m}=\sum_{i \in F} y_{i}=\left(a_{0} \bmod 1\right)$ in this case. In order to assign subsets to the connections $i j$ for a fixed customer $j$, we 'open' the cycle at a facility with maximum $c_{i j}$ yielding a line and construct the sets $I_{i j}$ again as in the proof of Theorem 4.2 (using the same assumption on the optimum LP solution). The resulting sets $I_{i}$ and $I_{i j}$ fulfill the conditions of Lemma 4.1 and the result follows.

The rounding technique in the proof of Theorem 4.3 has been applied before by Bertsimas, Teo, and Vohra (1999) to prove the integrality of an LP relaxation of the $k$-median problem on cycles. The insight from Theorem 4.3 can be used to prove the following result.

Corollary 4.4. The unconstrained facility location problem with unimodal connection costs on a cycle can be solved in polynomial time.

Proof. We add the constraint $\sum_{i} y_{i} \in \mathbb{Z}$ to the LP relaxation. It follows from the proof of Theorem 4.3 that an optimal solution to this stronger relaxation can be turned into an integral optimal solution in polynomial time. Moreover, the stronger relaxation can be solved in the following way: let $\gamma:=\sum_{i} y_{i}^{*}$ for an optimum solution of the original (LP). Then there exists an optimal solution to the stronger relaxation with $\sum_{i} y_{i} \in\{\lceil\gamma\rceil,\lfloor\gamma\rfloor\}$; this follows from the fact that the optimal value of the parametric linear program which we get by adding the constraint $\sum_{i} y_{i}=\mu$ to (LP) is a convex function of $\mu$, see, e.g., (Padberg 1995, Section 6.5). Therefore we only need to solve this relaxation for the two values $\mu=\lceil\gamma\rceil$ and $\mu=\lfloor\gamma\rfloor$ and take the better of the two solutions.

An alternative proof of Corollary 4.4 is based on the following observation. As we already mentioned in the introduction, the unconstrained facility location problem can be formulated as a covering problem (see Kolen and Tamir (1990)). Since the connection costs are unimodal, the rows in the linear programming formulation of this covering problem fulfill the circular 1's property (for definition, see Ahuja, Magnanti, and Orlin (1993, Chapter 9, Exercise 9.9)). If we add the constraint $\sum_{i} y_{i}=p$ for some $p \in \mathbb{N}$ ( $p$ can for example be determined by binary search), it is an easy exercise to show that an integral optimal solution exists since the matrix of the linear program is totally unimodular, see Ahuja, Magnanti, and Orlin (1993, Chapter 9, Exercise 9.9).

The above discussion has also implications for the $p$-median problem, where the number of facilities to open is a given integral number $p$. The proof of Theorem 4.2 shows that the corresponding LP relaxation (with the inequality $\sum_{i \in F} y_{i}=p$ ) has an integral optimum solution for instances defined on a cycle with unimodal costs; a generalization of a result first derived by Oudjit (1981) (for linear costs).

\subsection{Facility location on a tree}

In the following we consider unconstrained facility location problems on trees where all customers and facilities are located on the vertices of an acyclic connected graph. As for the case of a line, we assume that the connection $\operatorname{costs} c_{i j}$ of each customer $j$ are monotone such that $c_{i j} \leqslant c_{i^{\prime} j}$ if facility $i$ lies on the unique path between $j$ and facility $i^{\prime}$. Unfortunately, this condition is not sufficient to guarantee a non-empty core. The counterexample in Figure 5 consists of three customers and three facilities. The fixed cost of each facility is 1 and each customer can be connected to the facility at the same vertex of the tree or to its anti-clockwise neighbor for free; a connection to its clockwise neighbor, however, costs a large amount $M$. An optimum solution opens two of the three facilities and has value 2. In an optimum LP solution, however, each facility is opened with fraction $1 / 2$ resulting in total cost $3 / 2$. Thus, only a fraction of $3 / 4$ of the total cost can be recovered.

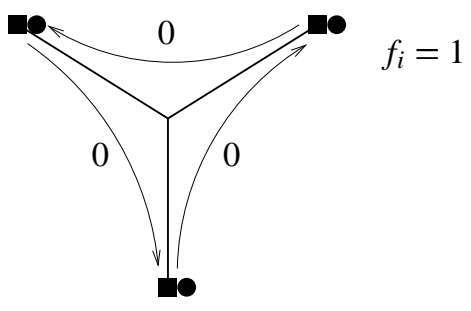

Figure 5: An instance of the unconstrained facility location problem on a tree with monotone connection costs but empty core. 
In the following we restrict to a special class of monotone connection costs on a tree: Let $d: E$ ! $\rightarrow \mathbb{R}^{\dagger}$ be a distance function on the edges of the tree. We denote the length of the unique path between two vertices $i$ and $j$ of the tree by $d_{i j}$.

Theorem 4.5 (Trubin (1976), Kolen (1983)). If for each customer $j$ the costs $c_{i j}$ for connecting $j$ to facilities $i \in F$ is an arbitrary nondecreasing function (possibly dependent on $j$ ) of the distances $d_{i j}$ in an underlying tree metric, then there is no integrality gap for the unconstrained facility location problem and the core is non-empty.

Using the same technique as in the proofs of Theorem 4.2 and Lemma 4.1, one can show that, for the class of instances under consideration, any feasible solution to the LP relaxation can be written as a convex combination of integral solutions. In particular, all vertices of the underlying polyhedron are integral. This result also follows from the work of Trubin (1976) (see also Grishukhin (1994)), Kolen (1983) (see also Kolen and Tamir (1990)) or Bárány, Edmonds, and Wolsey (1986) (see also Cornuéjols, Nemhauser, and Wolsey (1990)). They use a simple reduction of the unconstrained facility location problem to the set cover problem. For the class of instances considered in Theorem 4.5, the constraint matrix of the resulting set cover problem is totally balanced which yields the integrality result. Moreover, Trubin and also Kolen give an $O\left((|N|+|F|)^{3}\right)$ algorithm for solving such instances. This result has been improved by Gimadi (1983) to running time $O\left((|N|+|F|)^{2}\right)$. Furthermore, Ageev (1992) and Granot and Skorin-Karpov (1994) gave a polynomial-time algorithm for solving the unconstrained facility location problem on partial $k$-trees for fixed $k$.

Tamir (1992) considers a variant of the facility location problem on a tree where each customer $j$ has to be connected to a facility within a given distance $r_{j}$. This constraint can be modeled by letting $c_{i j}=0$ if the distance between customer $j$ and facility $i$ is at most $r_{j}$ and $c_{i j}=\infty$, otherwise; thus it is a special case of the problem discussed above. Tamir proves the result given in Theorem 4.5 for this special case.

Proof of Theorem 4.5. We construct sets $I_{i}$ and $I_{i j}$ that fulfill the conditions in Lemma 4.1. To simplify the presentation of the construction, we assume that there is at least one customer at each node of the tree; otherwise we can, without loss of generality, add dummy customers $j$ with $c_{i j}=0$ for all $i \in F$. For each customer $j$ we order the set of facilities $F$ by nondecreasing distances $d_{i j}$ and break ties according to increasing indices $i=1, \ldots, m$. Using the same arguments given in the proof of Theorem 4.2, we can restrict to LP solutions with the following property: If facility $i$ is closer to customer $j$ than facility $i^{\prime}$ (with respect to the above ordering) and $x_{i^{\prime} j}>0$, then $x_{i j}=y_{i}$. As an immediate consequence of this we get the following property $(*)$ : If customer $k$ lies on the unique path from customer $j$ to facility $i$, then $x_{i j} \leqslant x_{i k}$. In particular, if two customers $j$ and $j^{\prime}$ are located at the same node of the tree, we get $x_{i j}=x_{i j^{\prime}}$ for all $i \in F$. Therefore, all customers $j$ located at the same node will get the same sets $I_{i j}$ and we will only consider one customer per node in the following construction.

To assign subsets $I_{i}$ and $I_{i j}$ to the facilities and connections, respectively, we traverse the nodes of the tree in such a way that the first $k$ visited nodes form a subtree for any $k$. This can, e. g., be done by rooting the tree at some node and traversing the nodes of the tree by depth- or breadth-first search or in any order such that the predecessor of any node is visited before the node itself. During the algorithm we preserve the following invariant (**):

For all customers $j$ at visited nodes of the tree, the subsets $I_{i j}, i \in F$, form a partition of the interval $[0,1)$ such that $\left|I_{i j}\right|=x_{i j}$ and $I_{i j} \subseteq I_{i}$. Moreover, for all $i \in F$, there exists a visited node $j$ such that $I_{i j}=I_{i}$. for all $i \in F$.

In particular, this implies that $\left|I_{i}\right| \leqslant y_{i}$.

For the customer $j$ at the root of the tree, we set $I_{i}:=I_{i j}:=\left[\sum_{k=1}^{i-1} x_{i k}, \sum_{k=1}^{i} x_{i k}\right) \subseteq[0,1)$ for $i=1, \ldots, m$. Notice that the invariant $(* *)$ is fulfilled after this step. When we arrive at a node with a customer $j$, we denote the customer located at the predecessor of the current node in the tree by $k$. The following observation is crucial for the assignment of the subsets $I_{i}$ and $I_{i j}$.

Claim 1. For all $i \in F$, either $x_{i j} \leqslant x_{i k}$ or $x_{i k}=\left|I_{i}\right|$.

We postpone the proof of this claim after the complete description of the assignment procedure.

We first consider all facilities $i \in F$ with $x_{i j} \leqslant x_{i k}$ and choose an arbitrary subset $I_{i j} \subseteq I_{i k}$ of measure $x_{i j}$. For all the other facilities $i \in F$, namely those for which $x_{i j}>x_{i k}=\left|I_{i}\right|$ by Claim 1, choose arbitrary sets $I_{i j} \supset I_{i k}$ of measure $x_{i j}$ such that the sets $I_{i j}, i \in F$, form a partition of $[0,1)$ (this is possible since the sets $I_{i k}, i \in F$, form a partition) and redefine $I_{i}:=I_{i j}$. Notice that the invariant $(* *)$ is still fulfilled after this step.

Thus, the final sets $I_{i}$ and $I_{i j}$ fulfill the conditions of Lemma 4.1 and the result follows. 
Proof of Claim 1. Assume that $x_{i k}<x_{i j}$ and $x_{i k}<\left|I_{i}\right|$ (since $k$ has been visited). By the invariant (**), there exists a customer $h \neq k$ with $I_{i h}=I_{i}$, implying that $x_{i k}<x_{i h}$. By property $(*), k$ can neither lie on the path from $j$ to $i$ nor on the path from $h$ to $i$. However, since $k$ is the predecessor of $j$ in the tree and $h$ was visited, $k$ lies on the path between $j$ and $h-$ a contradiction.

\section{On the complexity of core computations}

In this section we prove the following theorem which confirms a conjecture by Chardaire (1998).

Theorem 5.1. For general instances of the unconstrained facility location problem it is NP-complete to decide whether the core is non-empty.

Proof. By Theorem 2.1 it suffices to show that it is NP-complete to decide whether or not the LP relaxation given in Section 2 has an integral optimal solution. The problem is obviously in NP. To prove that it is NP-hard, we use a reduction from $3 \mathrm{SAT}$. We restrict to instances where each clause contains exactly three (not necessarily different) literals.

Given an instance of 3SAT we construct the following facility location problem. For each variable $X$ we introduce one customer $j_{X}$ and two facilities $i_{X}$ and $i_{\bar{X}}$ corresponding to the two literals $X$ and its negation $\bar{X}$. The cost for connecting customer $j_{X}$ to these two facilities is 0 , the cost for opening one of the facilities is equal to 1 plus the number of occurrences of the corresponding literal in the instance.

For each clause $C$ we introduce one customer $j_{C}$ and for each of its three literals $L$ a facility $i_{C L}$ and a customer $j_{C L}$. Customer $j_{C}$ can be connected to the three facilities at cost 0 , the cost for opening each facility is 1 ; each customer $j_{C L}$ can be connected either to $i_{C L}$ or to $i_{L}$ at cost 0 . Finally, we introduce one additional dummy facility $i_{0}$ with fixed cost 0 . The customers $j_{C L}$ of all clauses can connect at cost 1 to this facility. An illustrating example of this construction is given in Figure 6. All connections that are not depicted in the figure have infinite cost.

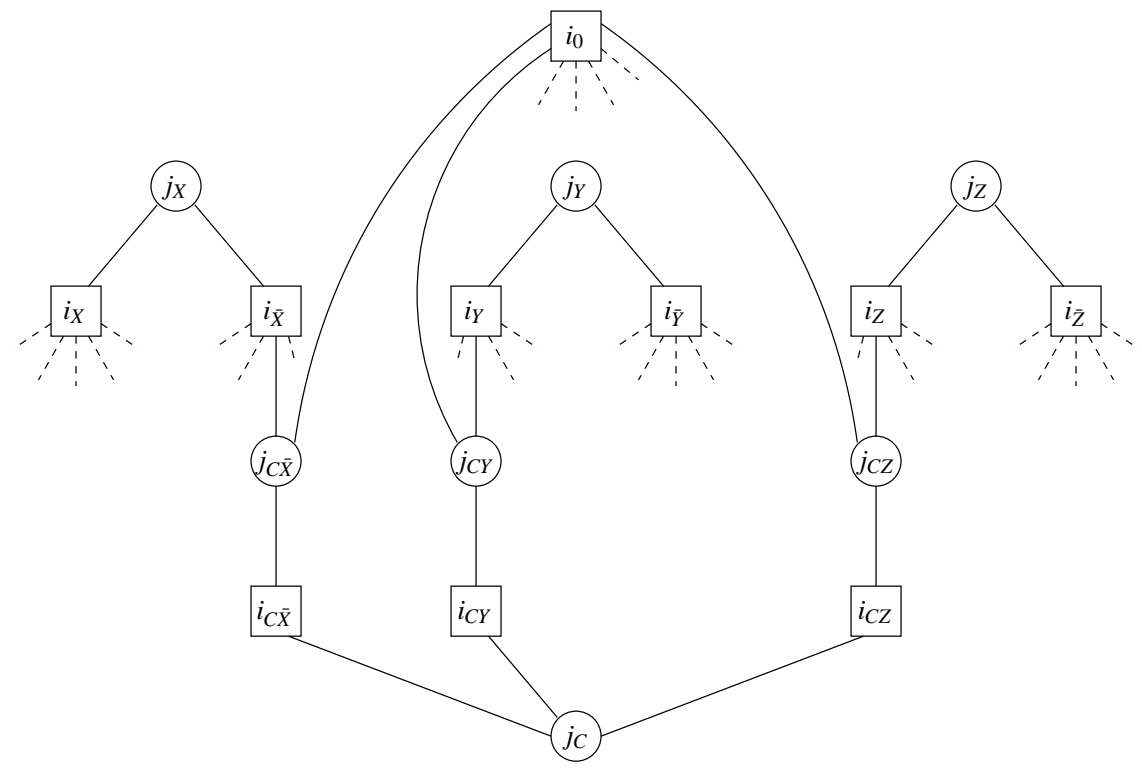

Figure 6: A facility location instance corresponding to an instance of 3S AT containing variables $X, Y$, and $Z$ and a clause $C=(\bar{X} \vee Y \vee Z)$.

We claim that the LP relaxation of this facility location instance has an integral optimal solution if and only if the underlying 3SAT instance can be satisfied. We first show that the optimal LP value is $n+3 m$ where $n$ denotes the number of variables and $m$ the number of clauses of the 3SAT instance. A feasible solution of value $n+3 m$ is 
given by

$$
\left.\begin{array}{rl}
y_{i_{L}} & =1 / 2 \\
x_{i_{X} j_{X}}=x_{i_{\bar{X}} j_{X}} & =1 / 2 \\
y_{i_{C L}}=x_{i_{C L} j_{C}}=x_{i_{C L} j_{C L}} & =1 / 3 \\
x_{i_{L} j_{C L}} & =1 / 2 \\
y_{i_{0}}=x_{i_{0} j_{C L}} & =1 / 6
\end{array}\right\} \quad \begin{aligned}
& \text { for each literal } L, \\
& \text { for each variable } X,
\end{aligned}
$$

In order to show that this solution is optimal, we construct a dual solution of value $n+3 m$. The dual of the LP relaxation is given by

$$
\begin{array}{cll}
\text { maximize } & \sum_{j \in N} v_{j} & \\
\text { subject to } & \sum_{j \in N} w_{i j}=f_{i} & \text { for all } i \in F, \\
& v_{j}-w_{i j} \leqslant c_{i j} & \text { for all } i \in F, j \in N, \\
& w_{i j} \geqslant 0 & \text { for all } i \in F, j \in N .
\end{array}
$$

A feasible dual solution of value $n+3 m$ is given by

$$
\left.\begin{array}{rl}
v_{j_{X}}=w_{i_{X} j_{X}}=w_{i_{\bar{X}} j_{X}}=1 & \text { for each variable } X, \\
v_{j_{C}}=w_{i_{C L} j_{C}}=w_{i_{0} j_{C L}}=0 \\
v_{j_{C L}}=w_{i_{C L} j_{C L}}=w_{i_{L} j_{C L}}=1
\end{array}\right\} \quad \begin{aligned}
& \text { for each clause } C \text { and each of its literals } L,
\end{aligned}
$$

Since the given dual solution is optimal, every primal optimal solution has to fulfill the following complementary slackness conditions:

$$
\begin{aligned}
& x_{i_{X} j_{X}}=y_{i_{X}} \quad x_{i_{\bar{X}} j_{X}}=y_{i_{\bar{X}}} \quad \text { for each variable } X, \\
& x_{i_{C L} j_{C L}}=y_{i_{C L}} \quad x_{i_{L} j_{C L}}=y_{i_{L}} \quad \text { for each clause } C \text { and each of its literals } L \text {. }
\end{aligned}
$$

Using these conditions we can show that any primal integral optimal solution $(x, y)$ corresponds to a satisfying truth-assignment of the underlying 3S AT instance. Condition (11) and constraint (10) yield that for each variable $X$ exactly one of the facilities $i_{X}$ and $i_{\bar{X}}$ has to be open. We set the variable $X$ to the value true if $y_{i_{X}}=0$ and to the value false if $y_{i_{\bar{X}}}=0$. For an arbitrary clause $C$, at least one of the three facilities $i_{C L}$ corresponding to the three literals of $C$ must be open to serve customer $j_{C}$. Condition (12) yields that the customer $j_{C L}$ is connected to this facility. It follows again from (12) that the facility $i_{L}$ is closed and the literal $L$ is thus set to the value true such that the clause $C$ is fulfilled.

Using the same interpretation one can easily show that any satisfying truth-assignment yields an integral optimal solution of the LP relaxation. This completes the proof.

We can state the following interesting corollary of the result in Theorem 5.1.

Corollary 5.2. Given an instance of the unconstrained facility location problem and a cost allocation vector $v \in \mathbb{R}^{N}$, it is NP-complete to decide whether or not $v$ is in the core of the corresponding game.

Proof. The problem is in NP since a positive answer to the question can be proven in the following way: Give an integral optimal solution to the LP relaxation and a completion $w$ of the given vector $v$ to an optimal dual solution $(v, w)$.

Quite interestingly, given the information that the core is non-empty, it is easy to compute an element of the core and to decide whether a given cost allocation $v$ belongs to the core. Both problems reduce to solving the dual of the LP relaxation of the problem.

Corollary 5.3. If the core of an unconstrained facility location game is non-empty, an element of the core can be computed in polynomial time and it can be checked in polynomial time whether a given cost allocation $v \in \mathbb{R}^{N}$ belongs to the core. 
It follows from the considerations in Section 3 that the result in Corollary 5.3 can be generalized to constrained variants of the facility location problem for which we can optimize over the discrete sets $P_{i}$ in polynomial time.

Acknowledgments. The authors are much indebted to Michel Le Breton for motivating them to study facility location problems from a game-theoretic point of view and for numerous interesting and helpful discussions on this subject. They would also like to thank Alexander Ageev and Maxim Sviridenko for pointing out the connection to totally balanced matrices mentioned in Subsection 4.3. Further helpful comments and pointers to the literature were given by two anonymous referees.

\section{References}

Ageev, A. A. (1983). On the complexity of minimization problems for polynomials in boolean variables. Upravlyaemye Sistemy 23, 3 - 11. (Russian).

Ageev, A. A. (1992). A criterion of polynomial-time solvability for the network location problem. In E. Balas, G. Cornuéjols, and R. Kannan (Eds.), Integer Programming and Combinatorial Optimization, pp. 237 245. Carnegie Mellon University, Pittsburgh.

Ahuja, R. K., T. L. Magnanti, and J. B. Orlin (1993). Network Flows. Theory, Algorithms, and Applications. Prentice Hall, Englewood Cliffs, NJ.

Balinski, M. L. (1965). Integer programming: Methods, uses, computation. Management Science 12, 253 - 313.

Bar-Noy, A., S. Guha, J. Naor, and B. Schieber (1999). Approximating the throughput of multiple machines under real-time scheduling. In Proceedings of the 31st Annual ACM Symposium on Theory of Computing, pp. $622-631$.

Bárány, I., J. Edmonds, and L. A. Wolsey (1986). Packing and covering a tree by subtrees. Combinatorica 6, $221-233$.

Bertsimas, D., C. Teo, and R. Vohra (1999). On dependent randomized rounding algorithms. Operations Research Letters 24, $105-114$.

Chardaire, P. (1998). Facility location optimization and cooperative games. Ph. D. thesis, University of East Anglia, Norwich, United Kingdom.

Chudak, F. A. (1998). Improved approximation algorithms for uncapacitated facility location. In R. E. Bixby, E. A. Boyd, and R. Z. Ríos-Mercado (Eds.), Integer Programming and Combinatorial Optimization, Volume 1412 of Lecture Notes in Computer Science, pp. 180 - 194. Springer, Berlin.

Cornuéjols, G., G. L. Nemhauser, and L. A. Wolsey (1990). The uncapacitated facility location problem. In P. Mirchandani and R. Francis (Eds.), Discrete Location Theory, pp. 119 - 171. John Wiley and Sons, Inc., New York.

Deng, X., T. Ibaraki, and H. Nagamochi (1999). Algorithmic aspects of the core of combinatorial optimization games. Mathematics of Operations Research 24, 751 - 766. Also in proceedings of the 8th Annual ACMSIAM Symposium on Discrete Algorithms, 1997.

Deng, X., T. Ibaraki, H. Nagamochi, and W. Zang (2000). Totally balanced combinatorial optimization games. Mathematical Programming 87, 441 - 452.

Deng, X. and C. H. Papadimitriou (1994). On the complexity of cooperative solution concepts. Mathematics of Operations Research 19, 257 - 266.

Faigle, U., S. P. Fekete, W. Hochstättler, and W. Kern (1997). On the complexity of testing membership in the core of min-cost spanning tree games. International Journal of Game Theory 26, 361 - 366.

Faigle, U. and W. Kern (2000). On the core of ordered submodular cost games. Mathematical Programming 87, $483-499$.

Gimadi, E. K. (1983). An effective algorithm for the solution of the location problem with service domains connected with respect to an acyclic network. (russian). Upravljaemye sistemy 23, $12-23$.

Göthe-Lundgren, M., K. Jörnsten, and P. Värbrand (1996). On the nucleolus of the basic vehicle routing game. Mathematical Programming 72, 83 - 100. 
Granot, D. and D. Skorin-Karpov (1994). On some optimization problems on $k$-trees and partial $k$-trees. Discrete Applied Mathematics 48, 129 - 145.

Grishukhin, V. P. (1994). On polynomial solvability conditions for the simplest plant location problem. Translations American Mathematical Society, Ser. 2 158, 37 - 46. Translation from Economico-mathematical modelling of discrete processes, Moscow, $52-65,1988$.

Grötschel, M., L. Lovász, and A. Schrijver (1988). Geometric Algorithms and Combinatorial Optimization, Volume 2 of Algorithms and Combinatorics. Springer, Berlin.

Guha, S. and S. Khuller (1998). Greedy strikes back: Improved facility location algorithms. In Proceedings of the 9th Annual ACM-SIAM Symposium on Discrete Algorithms, pp. 649 - 657.

Kolen, A. (1983). Solving covering problems and the uncapacitated plant location problem on trees. European Journal of Operational Research 12, $266-278$.

Kolen, A. and A. Tamir (1990). Covering problems. In P. Mirchandani and R. Francis (Eds.), Discrete Location Theory, pp. 263 - 304. John Wiley and Sons, Inc., New York.

Krarup, J. and O. Bilde (1977). Plant location, set covering and economic lot size: an $O(m n)$-algorithm for structured problems. In L. Collatz and W. Wetterling (Eds.), Numerische Methoden bei Optimierungsaufgaben Band 3 (Optimierung bei graphentheoretischen und ganzzahligen Problemen), Volume 36 of International series of numerical mathematics, pp. 155 - 180. Birkhaeuser Verlag.

Lenstra, J., D. Shmoys, and E. Tardos (1990). Approximation algorithms for scheduling unrelated parallel machines. Mathematical Programming 46, 259-271.

Mirchandani, P. B. and P. L. Francis (Eds.) (1990). Discrete Location Theory. John Wiley \& Sons.

Motwani, R. and P. Raghavan (1995). Randomized Algorithms. Cambridge University Press.

Moulin, H. (1995). Cooperative Microeconomics: A Game-Theoretic Introduction. Princeton University Press.

Nemhauser, G. L. and L. A. Wolsey (1988). Integer and Combinatorial Optimization. New York: John Wiley \& Sons.

Oudjit, A. (1981). Median Locations on Deterministic and Probabilistic Multidimensional Networks. Ph. D. thesis, Rensselaer Polytechnic Institute, Troy, New York.

Owen, G. (1995). Game Theory. Academic Press.

Padberg, M. (1995). Linear Optimization and Extensions. Springer, Berlin.

Samet, D. and E. Zemel (1984). On the core and dual set of linear programming games. Mathematics of Operations Research 9, $309-316$.

Sharkey, W. W. (1990). Cores of games with fixed costs and shared facilities. International Economic Review 31, $245-262$.

Shmoys, D. B. and E. Tardos (1993). An approximation algorithm for the generalized assignment problem. Mathematical Programming 62, $461-474$.

Tamir, A. (1992). On the core of cost allocation games defined on location problems. Transportation Science 27, $81-86$. Also in proceedings of the International Symposium on Locational Decisions, Skodsborg, Denmark, 1981.

Trubin, V. A. (1976). An effective algorithm for solving the distribution problem in a network in the form of a tree. Soviet Mathematics Doklady 17, 1604 - 1608. Translation from Doklady Akademija Nauk SSSR, 231:547 - 550, 1976.

Vazirani, V. (1999). Approximation algorithms. Unpublished manuscript. 